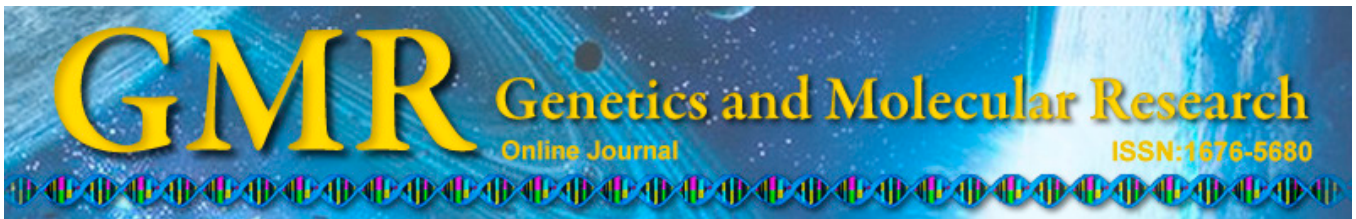

\title{
Effect of melatonin on oncosis of myocardial cells in the myocardial ischemia/reperfusion injury rat and the role of the mitochondrial permeability transition pore
}

L.F. Liu, Z.H. Qian, Q. Qin, M. Shi, H. Zhang, X.M. Tao and W.P. Zhu

Department of Gynecology and Obstetrics,

The Second Affiliated Hospital of Soochow University, Suzhou, China

Corresponding author: W.P. Zhu

E-mail: weipeizhu@yeah.net

Genet. Mol. Res. 14 (3): 7481-7489 (2015)

Received October 22, 2014

Accepted February 26, 2015

Published July 3, 2015

DOI http://dx.doi.org/10.4238/2015.July.3.24

\begin{abstract}
We aimed to evaluate the effect of melatonin on myocardial cell oncosis in the myocardial ischemia/reperfusion injury rat, and the role of the mitochondrial permeability transition pore (MPTP) therein. Sprague Dawley rats $(\mathrm{N}=60)$ were randomly divided into five groups of 12 rats each: control, ischemia/reperfusion (I/R), melatonin treatment (MT), melatonin treatment + atractyloside (MT+ATR), and atractyloside (ATR). We prepared the myocardial ischemia/reperfusion model by reperfusion after the left anterior descending coronary artery was ligated for $30 \mathrm{~min}$. The MT rats were given a $10 \mathrm{mg} / \mathrm{kg}$ MT intravenous injection immediately thereafter; the MT+ATR rats were also given a $5 \mathrm{mg} / \mathrm{kg}$ ATR intravenous injection $15 \mathrm{~min}$ before the ischemia; the ATR rats were given the ATR injection only. After 2-h reperfusion, myocardial tissue was extracted, the infarction size was determined, and myocardial ultrastructures were observed using electron microscopy. The expression level of the pre-oncosis receptor (porimin),
\end{abstract}


which can induce membrane injury, was determined by western blot; the nicotinamide adenine dinucleotide $\left(\mathrm{NAD}^{+}\right)$content was determined spectrophotometrically. The four treatment groups showed upregulated expression of myocardial porimin, increased myocardial infarction size, and reduced $\mathrm{NAD}^{+}$content $(\mathrm{P}<0.05)$. Compared with the $\mathrm{I} / \mathrm{R}$ and MT+ATR groups, MT rats showed downregulated expression of myocardial porimin, reduced myocardial infarct size, and increased myocardial cell NAD ${ }^{+}$content $(\mathrm{P}<0.05)$. The above indices between the ATR and MT+ATR groups were not significantly different $(\mathrm{P}>0.05)$. Thus, MT might protect myocardial ischemia/reperfusion rats by inhibiting MPTP opening and reducing myocardial cell oncosis.

Key words: Melatonin; Myocardial ischemia/reperfusion injury; Mitochondria

\section{INTRODUCTION}

Ischemia/reperfusion injury after myocardial infarction is a difficult problem, which clinicians have to face. Oncosis and apoptosis are the main manner of death for cells during myocardial ischemia/reperfusion injury (Buja, 2005). Cellular oncosis is a form of cell death different from apoptosis, and is characterized by cell swelling and karyolysis (Dacheux et al., 2000; Senthamizhselvan et al., 2014). The morphological characteristics of oncosis include an increase in the permeability of the cell membrane, a damage of integrity, and karyolysis accompanied by an inflammatory reaction. The mitochondrial permeability transition pore (MPTP) is a non-specific channel between the inner and outer membranes of mitochondria. The opening of the MPTP is widely considered to be closely related with ischemia/reperfusion injury of myocardial cells, and is currently considered a primary effector of myocardial ischemia/reperfusion injury; in addition, the MPTP plays an important role in the cell death pathway (Wang et al., 2014). Many studies have shown that melatonin treatment (MT) had a protective effect on myocardial ischemia/reperfusion injury (Husainy et al., 2012), but the specific mechanism has not been fully elucidated. Whether the mechanism of myocardial protection is related to the inhibition of myocardial cell oncosis by MT needs to be investigated, as well as the role of MPTP therein. This goal of this study was to evaluate the effect of MT on myocardial cell oncosis in the myocardial ischemia/reperfusion injury rat and the potential role of MPTP in this process.

\section{MATERIAL AND METHODS}

\section{Establishment of the animal model and grouping}

We obtained 60 healthy, clean-grade adult male Sprague Dawley rats of three months of age and body weight 280-360 g from the Experimental Animal Center of Soochow University School of Medicine, Suzhou, China. By using a random number table method, the rats were randomly divided into five groups $(\mathrm{N}=12)$ : controls $(\mathrm{CON})$, ischemia/reperfusion $(\mathrm{I} / \mathrm{R})$, MT, MT + atractyloside (MT+ATR), and ATR only. This study was carried out in strict accordance with the recommendations in the Guide for the Care and Use of Laboratory Animals, 
eighth edition, 2010, of the National Institutes of Health (Bethesda, MD, USA). The animal use protocol was reviewed and approved by the Institutional Animal Care and Use Committee of the Second Affiliated Hospital of Soochow University.

The rats were anaesthetized by intraperitoneal injection of $50 \mathrm{mg} / \mathrm{kg}$ pentobarbital sodium. A catheter filled with heparin was implanted in the right internal jugular vein and internal jugular artery for intravenous administration, analysis of arterial blood gas, or monitoring arterial blood pressure. Each rat received a tracheotomy and an endotracheal tube was inserted. An ALC-V9 animal ventilator was connected for positive end expiratory pressure, and the concentration of inhaled oxygen was $33 \%$. The respiratory frequency and tidal volume were regulated to maintain the $\mathrm{pH}$ value at $7.35-7.45,25-40 \mathrm{mmHg}(1 \mathrm{mmHg}$

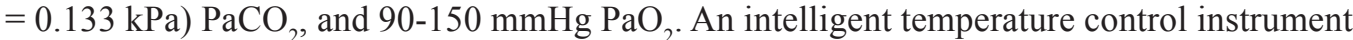
was used to maintain the body temperature of the rats at $36^{\circ}-37^{\circ} \mathrm{C}$. A thoracic incision in the left fifth intercostal space of the rat was made, and the pericardium was opened. The left anterior descending coronary artery (LAD) was ligated at the lower edge of left auricle with a 6-0 scatheless suture line. The suture end was crossed over the self-made ring sleeve to balance for $30 \mathrm{~min}$. The ring sleeve was used to block the blood supply of the LAD by hemostatic clamp. If the epicardium developed cyanosis or became pale and the electrocardiogram (ECG) showed transient arrhythmia and arched elevation of the ST segment, the signs were taken to suggest that ischemia was successful. The ring sleeve was subsequently loosened to perform reperfusion; epicardial recongestion was taken to prove successful reperfusion (Ceyran et al., 2008).

After the operation was completed, the rats remained stabilized for $30 \mathrm{~min}$. The CON rats only received threading but the LAD was not ligated; simple ischemia/reperfusion was implemented on the rats in the I/R group. After threading was completed, the rats remained stabilized for $30 \mathrm{~min}$. Subsequently, the LAD was blocked to cause ischemia for $30 \mathrm{~min}$. Then the LAD was opened for reperfusion for $2 \mathrm{~h}$. The rats in the MT group were given $10 \mathrm{mg} / \mathrm{kg}$ MT intravenously immediately after the $30 \mathrm{~min}$ ischemia period. The rats in the MT+ATR group were also given $5 \mathrm{mg} / \mathrm{kg}$ ATR intravenously $15 \mathrm{~min}$ before ischemia. The rats in the ATR group were only given the ATR intravenous injection 15 min before ischemia.

\section{Determination of myocardial infarction size}

The LADs in six rats were blocked again after reperfusion for $2 \mathrm{~h}$, and $2 \mathrm{~mL} \mathrm{1 \%} \mathrm{Evans}$ blue was injected in the internal jugular vein to blue-stain the normal region of the left ventricle (LV). The heart was quickly removed to separate the LV. The heart was transected into 5-6 tissue blocks with $2 \mathrm{~mm}$ thick using a tissue slicer. The blue stained normal tissue in the LV was isolated from the undyed ischemic tissue. According to the triphenyltetrazolium chloride (TTC) staining method, the myocardial tissue was placed in $0.5 \% \mathrm{TTC}$ in a water bath at $37^{\circ} \mathrm{C}$ for $15 \mathrm{~min}$, and the tissue was fixed with $10 \%$ formaldehyde overnight. The $\mathrm{LV}$ was divided into 3 parts including normal, ischemia non-infarction, and ischemia infarction areas (gray) under a microscope. The sections were weighed, and the myocardial infarction area was calculated (percentage of myocardial mass in the infarction area accounting for the myocardial mass in the ischemic area).

\section{Observation of myocardial ultrastructure}

The rat myocardial tissue was extracted after reperfusion for $2 \mathrm{~h}$; a 1 x 1 x $1 \mathrm{~mm} \mathrm{sec}-$ 
tion of tissue in the LV anterior wall was taken at ligature $3 \mathrm{~mm}$ toward the apical direction, placed in $4 \%$ glutaraldehyde to pre-fix for $3 \mathrm{~h}$, rinsed with $0.1 \mathrm{M}$ phosphate buffered saline, subjected to gradient dehydration, soaked and embedded with epoxy resin, sliced with an ultrathin microtome (to approximately $50 \mathrm{~nm}$ ), and finally double stained with uranyl acetatelead citrate. The myocardial ultrastructures were observed under an H-600 transmission electron microscope (Hitachi Ltd., Tokyo, Japan).

\section{Western blot}

After reperfusion for $2 \mathrm{~h}$, six rats were randomly chosen from which to obtain the hearts, and frozen at $80^{\circ} \mathrm{C}$ in liquid nitrogen. The lysate and phosphatase inhibitors were added to homogenize and crack in the low temperature under ultrasonic. The protein was extracted from the lysate, and the protein content in each sample was determined with a BCA protein concentration assay kit (Batch number: P0010S, Biyuntian Biotechnology Research Institute, Shanghai, China). The lysate was added to regulate to the similar concentration, continuously heated in a metal bath at $97^{\circ} \mathrm{C}$ for $5 \mathrm{~min}$. Twenty micrograms protein was extracted in each sample, protein was separated by $12 \%$ polyacrylamide gel electrophoresis (BioRad, Hercules, CA, USA). After electrophoresis, proteins were transferred to a nitrocellulose membrane (Millipore, Boston, MA, USA), and incubated for $2 \mathrm{~h}$ with $5 \%$ skim milk. The first antibody, inducing membrane injury pre-oncosis receptor (porimin, 1:1000, batch number: WH0114908M2, Sigma-Aldrich, St. Louis, MO, USA) and the internal reference GAPDH antibody (1:1000, batch number: AG019, Biyuntian Biotechnology Research Institute, Shanghai, China) were added respectively, and incubated overnight at $4^{\circ} \mathrm{C}$. The membrane was washed three times with Tris buffered saline/Tween 20 solution, and a secondary antibody was added with incubation for $2 \mathrm{~h}$ in a shaker. An ECL kit (Batch number: 34077, Thermo-Scientific, Rockford, IL, USA) was used for color development, and an enhanced chemiluminescence reaction was performed; the PVDF film was put into the luminous liquid (Merck KGaA, Darmstadt, Germany), transferred to the darkroom, developed, and fixed with automatic processing machine after exposure. The gray value of the bands was determined by using Image software. The ratio of the objective band gray value and the GAPDH gray value for each sample was used to reflect the expression level of myocardial porimin.

\section{Determination of the opening degree of MPTPs}

The nicotinamide adenine dinucleotide $\left(\mathrm{NAD}^{+}\right)$in the rat myocardial cells was extracted and the levels determined after reperfusion for $2 \mathrm{~h}$ in accordance with the method described in Wang et al. (2006), in order to reflect the MPTP opening number. The NAD content was inversely proportional to the MPTP opening number. To isolate $\mathrm{NAD}^{+}, 30 \mathrm{mg}$ left ventricular tissue was taken and ground in a glass homogenizer pre-refrigerated by liquid nitrogen. To this, $2 \mathrm{~mL} 0.6 \mathrm{M} \mathrm{HClO}_{4}$ was added to prepare the homogenate, of which $1 \mathrm{~mL}$ was centrifuged, and the supernatant extracted. In an ice bath, $0.2 \mathrm{~mL} 1 \mathrm{M} \mathrm{K}_{2} \mathrm{HPO}_{4}$ was added, followed by addition of $3 \mathrm{M} \mathrm{KOH}$ to neutralize the solution and maintain a $\mathrm{pH}$ value of 7.4. Following formation of the precipitate, the supernatant was extracted, and 20 $\mu \mathrm{L}$ alcohol dehydrogenase was added. The optical density value at $340 \mathrm{~nm}$ was determined using a DU 640 spectrophotometer (Beckman-Coulter, Miami, FL, USA), and the NAD ${ }^{+}$ content was calculated. 


\section{Statistical analysis}

The data were analyzed by the SAS 8.2 statistical software (SPSS Inc, Chicago, IL, USA). The measurement data are reported as means \pm standard deviation. The intra-group comparisons were expressed using analysis of variance of repeated measurement design. The comparisons between groups were expressed with single factor analysis of variance. $\mathrm{P}<0.05$ was taken to indicate that the difference was statistically significant.

\section{RESULTS}

\section{Comparison of the myocardial infarction sizes of the rats in each group}

As shown in Figure 1, compared with the CON group, the myocardial infarction sizes in the treatment groups increased significantly. Among them, the myocardial infarction size in the MT group decreased more than those in the other three groups $(\mathrm{P}<0.05)$. There was no significant difference between the ATR and MT+ATR groups $(\mathrm{P}>0.05)$.

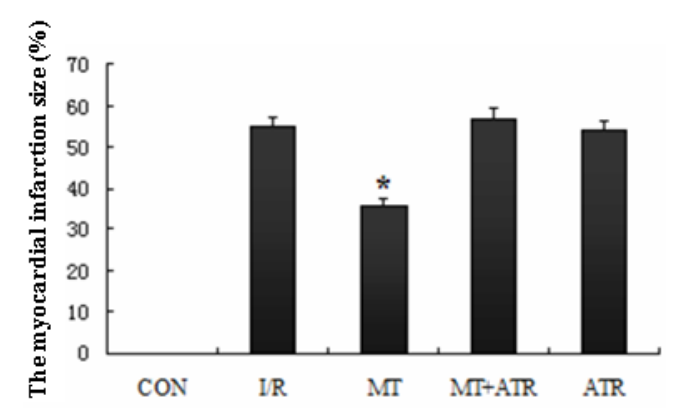

Figure 1. Comparison of myocardial infarction size of rats in each group.

\section{Ultrastructural changes of the rat myocardia in each group}

The myocardial cell structures in the CON group were clear, with regular arrangement of the myofibrils; each band was distinct, and the mitochondria appeared normal with uniform size and structural integrity. The myocardial cell structures in the MT group were clear, with irregularly arrayed myofibrils; every band of the sarcomere could be determined, and the mitochondrial structures were relatively fuzzy, with partial cristae loss. The myofibrils showed disorderly arrangements in the I/R, MT+ATR, and ATR groups; the myofilaments had dissolved, and the mitochondrial volumes were increased with vacuolization and obvious swelling; in addition, the mitochondrial crests had broadened, and mitochondria were ruptured with necrosis in the cytoplasm.

\section{Porimin expression in the rat myocardial cells}

The expression of porimin in the CON group was the lowest, while the expression levels of porimin in the myocardial cells in the treatment groups were significantly increased in comparison. Compared with that in the I/R, MT+ATR, and ATP groups, the expression of 
porimin in the myocardial cells in the MT group was decreased. There was no significant difference between the ATR and MT+ATR groups $(\mathrm{P}>0.05)$ (Figure 2).
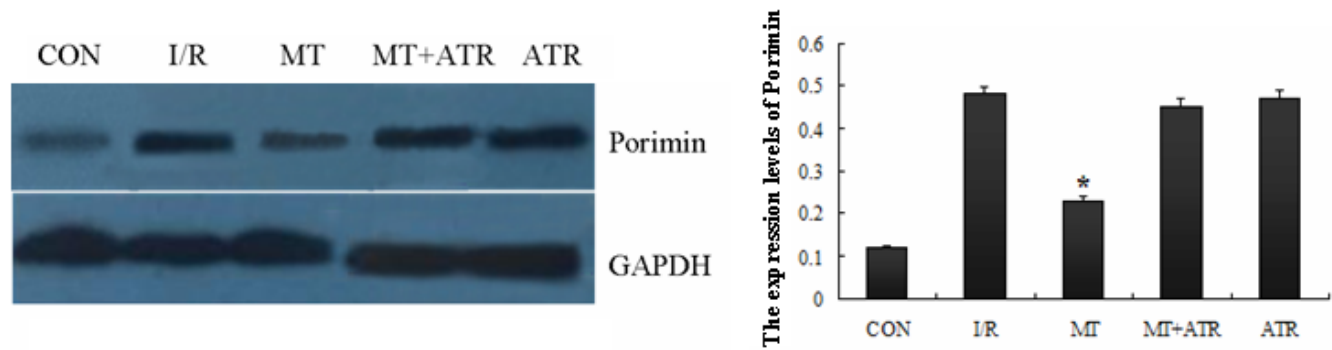

Figure 2. Expression of porimin in myocardial cells in each group.

\section{Degree of MPTP opening}

As shown in Figure 3, the myocardial $\mathrm{NAD}^{+}$contents in the $\mathrm{I} / \mathrm{R}, \mathrm{MT}, \mathrm{MT}+\mathrm{ATR}$, and ATP groups were significantly decreased compared with that in the CON group $(\mathrm{P}<0.05)$. The myocardial NAD ${ }^{+}$content in the MT group was increased significantly $(\mathrm{P}<0.05)$ compared with that in the I/R and MT+ATR groups. There was no significant difference between the ATR and MT+ATR groups $(\mathrm{P}>0.05)$.

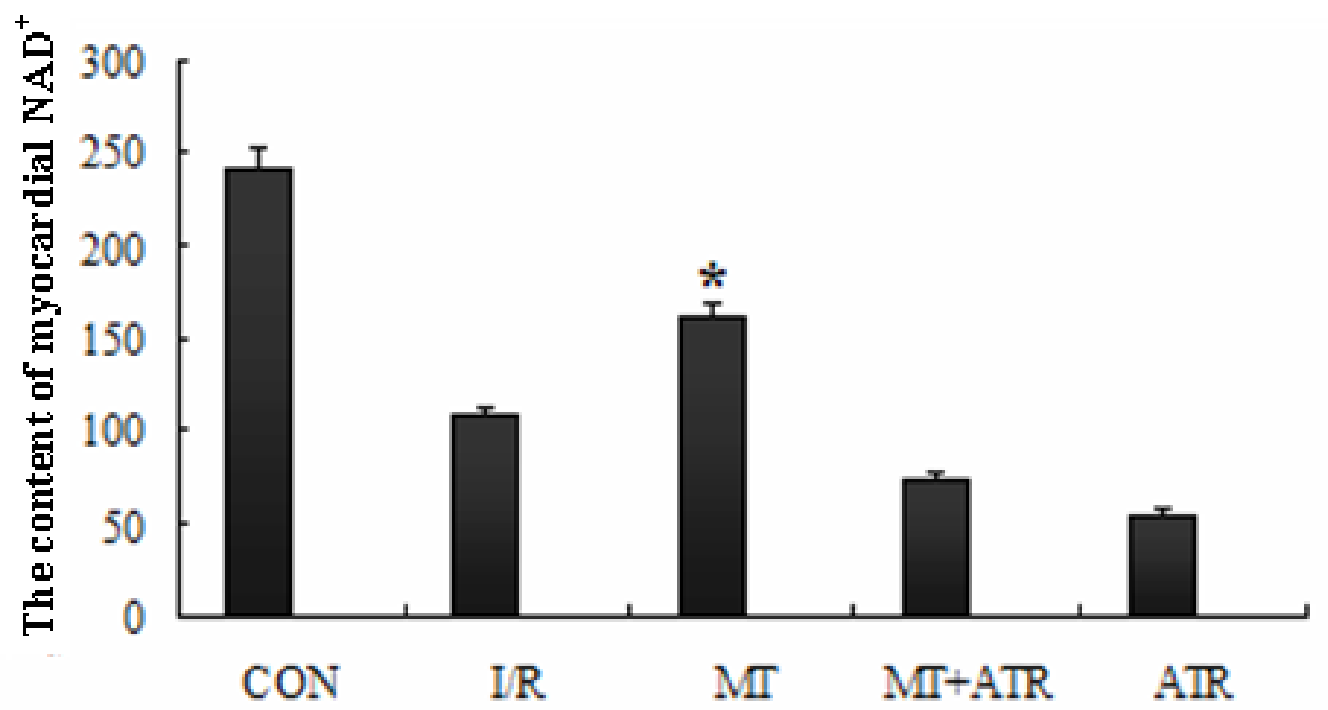

Figure 3. Comparison of the content of myocardial $\mathrm{NAD}^{+}$in each group.

\section{DISCUSSION}

Melatonin is a neuroendocrine hormone secreted by the pineal gland, and has extensive physiological activities and pharmacological functions. Studies have shown that mela- 
tonin could inhibit the production of a large number of free radicals during the myocardial ischemia/reperfusion period, and had a protective effect on myocardial ischemia/reperfusion. However, it remains to be determined whether other mechanisms exist through which melatonin protects the damaged myocardium. The study generated a myocardial ischemia/reperfusion injury model by blocking LAD to cause ischemia for $30 \mathrm{~min}$, according to published protocols (Zhu et al., 2011), followed by reperfusion recovery for $2 \mathrm{~h}$. The results showed that compared with the CON group, the myocardial infarction size in the I/R group significantly increased after reperfusion, and the pathological damage was aggravated, suggesting that the model was successful. This study selected the treatment dosage of ATR according that suggested by the literature (Yin et al., 2013). According to published report (Petrosillo et al., 2009) and preliminary experimental results, we implemented MT as well. Intravenous MT (10 $\mathrm{mg} / \mathrm{kg}$ ) was administered immediately after a 30 -min period of ischemia. The results showed that $10 \mathrm{mg} / \mathrm{kg}$ MT could produce an obvious myocardial protective effect: the mitochondrial $\mathrm{NAD}^{+}$content was rich, accounting for over $90 \%$ of tissue $\mathrm{NAD}^{+}$content. In the myocardial $\mathrm{I} / \mathrm{R}$ process, $\mathrm{NAD}^{+}$is released from mitochondria through the opening of MPTPs, penetrates the damaged cell membrane, and is washed out by reperfusion. Therefore, the $\mathrm{NAD}^{+}$content in myocardial tissue can reflect the opening degree of the MPTPs. Lower content reflects a higher MPTP opening degree and a heavier degree of myocardial damage (Yang et al., 2013).

The results of this study showed that compared with the I/R and MT+ATR groups, the expression of porimin was downregulated in the MT group. Combined with the results of the myocardial histopathology, this result suggested that MT can reduce the incidence of myocardial cell oncosis. A previous study found that the membrane specific receptor porimin, which can mediate oncosis, was present on the cell membrane; they also successfully cloned this receptor (Di Lisa et al., 2001). Porimin is a highly glycosylated transmembrane receptor protein containing 118 amino acids, and belongs to the cell membrane associated mucin family. The protein can specifically express on the surface of the cell in which oncosis is about to occur. Porimin can be activated in the case of anoxic conditions, lack of substrate, or progressively decreased ATP, and quickly binds its ligand, anti-porimin $\mathrm{mAb}$, resulting in assorted membrane structural damage, such as cell membrane bleb, the increase of cell membrane permeability, or the formation of pores; thus, the process of oncosis can be initiated. Oncosis in turn is an important component of the damage resultant from the myocardial ischemia/ reperfusion process.

The results of this study showed that after the MPTP opener ATR was administered, the expression of porimin in ischemia/reperfusion rats increased. In addition, the myocardial protective effects of MT treatment were found to be antagonistic to this effect, suggesting that MT treatment could protect rats from the consequences of myocardial ischemia/reperfusion by inhibiting the opening of MPTP and reducing myocardial cell oncosis. MPTP is a composite channel composed of multiple proteins located in the mitochondrial inner and outer membrane that regulates the mitochondrial membrane permeability. At the early stage of reperfusion, irreversible opening of MPTP allowed the passage of substances of molecular weight less than $1500 \mathrm{Da}$ through the mitochondrial inner membrane, causing the dissipation of the mitochondrial proton-electrochemical gradient, an irreversible decline of the mitochondrial transmembrane potential, disruption of ion homeostasis, mitochondrial swelling and rupture, and hydrolysis of ATP; in consequence, the level of intracellular ATP decreased, the level of cytoplasmic $\mathrm{Ca}^{2+}$ rose, and oncosis and apoptosis inducing factors were released, eventually leading to cell death (Li et al., 2014; Prendes et al., 2014). 
This study also found that both apoptosis and oncosis could be seen in the myocardial infarction in the apex under the electron microscope, although primarily oncosis cells were observed. Whether apoptosis or oncosis occurred was generally associated with stimulus intensity and action time. Weak intensity and short action time generally resulted in apoptosis; in contrast, strong stimulus intensity, long action time, and relatively serious cell injury led to death largely by oncosis. Research has shown that the level of intracellular ATP can also affect the mode of cell death. The cell choice of apoptosis or oncosis under the same stimulation depended on the level of intracellular ATP. If the energy demands could not be satisfied, the program of apoptosis was unable to be completed, and the cells would turn to oncosis. If ATP was supplemented, cell death would occur via apoptosis (Maslov et al., 2013). The MPTP opening degree directly affected the level of intracellular ATP, thus indirectly determining the mode of cell death. In summary, heavier I/R damage leads to a larger MPTP opening degree and a greater decrease of intracellular ATP, thus predisposing the cell towards oncosis. The results therefore suggest that MT reduces the occurrence of cell oncosis and the myocardial injury by inhibiting the opening of MPTPs.

In conclusion, this study demonstrated that MT can reduce myocardial cell oncosis and protect myocardial ischemia reperfusion in rats by inhibiting the opening of MPTPs.

\section{ACKNOWLEDGMENTS}

Research supported by the Young Workers Scientific Research Fund Project of the Second Affiliated Hospital of Soochow University (\#SDFEYQN1301).

\section{REFERENCES}

Buja LM (2005). Myocardial ischemia and reperfusion injury. Cardiovasc. Pathol. 14: 170-175.

Ceyran H, Narin F, Narin N, Akgun H, et al. (2008). The effect of high dose melatonin on cardiac ischemia- reperfusion injury. Yonsei Med. J. 49: 735-741.

Dacheux D, Toussaint B, Richard M, Brochier G, et al. (2000). Pseudomonas aeruginosa cystic fibrosis isolates induce rapid, type III secretion-dependent, oncosis of macrophages and polymorphonuclear neutrophils. Infect. Immun. 68: 2916-2924.

Di Lisa F, Menabò R, Canton M, Barile M, et al. (2001). Opening of the mitochondrial permeability transition pore causes depletion of mitochondrial and cytosolic $\mathrm{NAD}^{+}$and is a causative event in the death of myocytes in postischemic reperfusion of the heart. J. Biol. Chem. 276: 2571-2575.

Husainy MA, Dickenson JM and Galinanes M (2012). The MPTP status during early reoxygenation is critical for cardioprotection. J. Surg. Res. 174: 62-72.

Li Q, Guo HC, Maslov LN, Qiao XW, et al. (2014). Mitochondrial permeability transition pore plays a role in the cardioprotection of CB2 receptor against ischemia-reperfusion injury. Can. J. Physiol. Pharmacol. 92: 205-214.

Maslov LN, Naryzhnaia NV, Hanuš L, Pei JM, et al. (2013). Problem of end-effector of ischemic postconditioning of the heart. Ross. Fiziol. Zh. Im. I. M. Sechenova. 99: 555-574.

Petrosillo G, Colantuono G, Moro N, Ruggiero FM, et al. (2009). Melatonin protects against heart ischemia-reperfusion injury by inhibiting mitochondrial permeability transition pore opening. Am. J. Physiol. Heart Circ. Physiol. 297: H1487-1493.

Prendes MG, Hermann R, Torresin ME, Vélez D, et al. (2014). Role of mitochondrial permeability transition pore and mitochondrial ATP-sensitive potassium channels in the protective effects of ischemic preconditioning in isolated hearts from fed and fasted rats. J. Physiol. Biochem. 70: 791-800.

Senthamizhselvan O, Manivannan J, Silambarasan T and Raja B (2014). Diosmin pretreatment improves cardiac function and suppresses oxidative stress in rat heart after ischemia/reperfusion. Eur. J. Pharmacol. 736: 131-137.

Wang C, Weihrauch D, Schwabe DA, Bienengraeber M, et al. (2006). Extracellular signal-regulated kinases trigger isoflurane preconditioning concomitant with upregulation of hypoxia-inducible factor-1alpha and vascular endothelial growth factor expression in rats. Anesth. Analg. 103: 281-288. 
Wang Q, Sun Y, Li J, Xing W, et al. (2014). Quaternary ammonium salt of U50488H, a new א-opioid receptor agonist, protects rat heart against ischemia/reperfusion injury. Eur. J. Pharmacol. 15: 177-184.

Yang Y, Duan W, Jin Z, Yi W, et al. (2013). JAK2/STAT3 activation by melatonin attenuates the mitochondrial oxidative damage induced by myocardial ischemia/reperfusion injury. J. Pineal. Res. 55: 275-286.

Yin M, Wang C, Zhang J, Zhu J, et al. (2013). Effects of sevoflurane postconditioning on myocardial ischemia-reperfusioninduced oncosis of cardiomyocytes in rats: relationship with mitochondrial permeability transition pore. Chin. $J$. Anesthesiol. 33: 718-721.

Zhu J, Rebecchi MJ, Glass PS, Brink PR, et al. (2011). Cardioprotection of the aged rat heart by GSK-3beta inhibitor is attenuated: age-related changes in mitochondrial permeability transition pore modulation. Am. J. Physiol. Heart Circ Physiol. 300: H922-H930. 